\title{
Amaranto: distribución y diversidad morfológica del recurso genético en partes de la región Maya (sureste de México, Guatemala y Honduras)
}

\section{Amaranth: distribution and morphological diversity of the genetic resource in parts of the Mayan region (southeast of Mexico, Guatemala and Honduras)}

\author{
Erick A. Aguilera-Cauich' (iD), Karen Z. Solís-Fernández' (iD), Ariadna Ibarra-Morales' (iD), Rolando Cifuentes-Velásquez² (iD), \\ Ivonne Sánchez-del Pinol,3 (iD)
}

\section{Resumen:}

Antecedentes y Objetivos: El amaranto es de interés mundial por su valor nutricional como verdura y grano. Las especies cultivadas de mayor importancia comercial por su producción de grano son Amaranthus caudatus, A. cruentus y A. hypochondriacus. Dos hipótesis aceptadas sobre el origen de $A$. cruentus consideran a A. hybridus como su ancestro. Los objetivos del presente estudio son 1) reportar la distribución actualizada y frecuencia de $A$. cruentus y $A$. hybridus en el sureste de México, Guatemala y Honduras y 2 ) evaluar su diversidad morfológica mediante caracteres florales y del fruto de ambas especies en México y Guatemala.

Métodos: La información proveniente de 147 ejemplares de herbario fue empleada para actualizar la distribución y frecuencia de $A$. cruentus y $A$. hybridus en la zona de estudio, posible área de domesticación. La diversidad a nivel morfológico se determinó del análisis de 22 caracteres relacionados a estructuras de flores pistiladas y del fruto, usando un análisis multivariado de componentes principales y evaluando para ello 15 ejemplares de $A$. cruentus y 31 de $A$. hybridus procedentes de Guatemala y el sureste de México.

Resultados clave: La mayor distribución de A. cruentus se presentó en Guatemala y, en el caso de $A$. hybridus en México. La variación acumulada en los tres primeros componentes principales fue de $58 \%$ para ambas especies. La variación morfológica para las dos especies se asoció principalmente con caracteres relacionados a los sépalos internos y externos. El gráfico del análisis de componentes principales permite observar una amplia variación morfológica en $A$. cruentus y $A$. hybridus, independiente de su distribución geográfica en ambos casos.

Conclusiones: La distribución geográfica de A. cruentus en México es más amplia con respecto a reportes previos. Se describe la diversidad morfológica de estructuras de flores pistiladas y frutos en $A$. cruentus y $A$. hybridus.

Palabras clave: Amaranthus cruentus, Amaranthus hybridus, centro de origen, diversidad morfológica.

\section{Abstract:}

Background and Aims: Amaranth is of worldwide interest for its nutritional value as a vegetable and grain. The principal cultivated species used as grain are Amaranthus caudatus, A. cruentus and A. hypochondriacus. Two accepted hypotheses about the origin of $A$. cruentus consider $A$. hybridus as its ancestor. The objectives of this study are 1 ) to report the updated distribution and frequency of $A$. cruentus and $A$. hybridus in southeastern Mexico, Guatemala, and Honduras and 2) to assess their morphological diversity, using floral and fruit characters of both species in Mexico and Guatemala. Methods: The information gathered from 147 herbarium examples was used to update the distribution and determine the frequency of $A$. cruentus and $A$. hybridus in the study area, possible area of domestication. Morphological diversity was determined using 22 morphological characters related to pistillate flowers and fruits by conducting a multivariate analysis of main components in a sampling of 15 specimens of $A$. cruentus and 31 of $A$. hybridus from Guatemala and southeastern Mexico.

Key results: The main distribution of $A$. cruentus was shown in Guatemala, and of $A$. hybridus in Mexico. The accumulated variation in the first three principal components was $58 \%$ for both species. The morphological variation for both species was mainly associated with characters related to the internal and external sepals. The principal components analysis graph showed wide morphological variation in $A$. cruentus and $A$. hybridus regardless of their geographical distribution in both cases.

Conclusions: The geographical distribution of $A$. cruentus in Mexico is wider than previously reported. Diversity was described using morphological characters of pistillate flowers and fruits for $A$. cruentus and $A$. hybridus.

Key words: Amaranthus cruentus, Amaranthus hybridus, center of origin, morphological diversity.

${ }^{1}$ Centro de Investigación Científica de Yucatán, A.C., Calle 43 No. $130 \times 32$ y 34, Col. Chuburná de Hidalgo, 97205 Yucatán, México.

${ }^{2}$ Universidad del Valle de Guatemala, Centro de Estudios Agrícolas y Alimentarios, 18 Av. 11-95 zona 15 Vista Hermosa III, 01015 Guatemala, Guatemala.

${ }^{3}$ Autor para la correspondencia: isanchez@cicy.mx
Recibido: 1 de junio de 2020.

Revisado: 25 de junio de 2020.

Aceptado por Marie-Stéphanie Samain: 31 de agosto de 2020.

Publicado Primero en línea: 22 de septiembre de 2020 Publicado: Acta Botanica Mexicana 128 (2021).

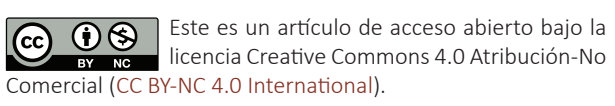

Citar como: Aguilera-Cauich, E. A., K. Z. Solís-Fernández, A. Ibarra-Morales, R. Cifuentes-Velásquez e I. Sánchez-del Pino. 2020(2021). Amaranto: distribución y diversidad morfológica del recurso genético en partes de la región Maya (sureste de México, Guatemala y Honduras). Acta Botanica Mexicana 128: e1738. DOI: https://doi.org/10.21829/abm128.2021.1738 


\section{Introducción}

El amaranto pertenece a la familia Amaranthaceae, subfamilia Amaranthoideae, género Amaranthus L. (Carmona y Orsini-Velázquez, 2010). Este género es predominantemente tropical, se distribuye en todos los climas desde el nivel del mar hasta 3600 m (Mazón et al., 2003). Borsch et al. (2015) reportan que la familia Amaranthaceae se conforma por 82 géneros y 840 especies. Costea et al. (2001) indican que aproximadamente 40 especies de Amaranthus son nativas de América.

El género Amaranthus se caracteriza por incluir plantas herbáceas anuales o arbustivas con flores de diversos colores, de verde a morado o púrpura con distintas coloraciones intermedias; unisexuales, con flores masculinas y femeninas en la misma planta (monoicas) o en plantas diferentes (dioicas) (Mosyakin y Robertson, 1996). Además de su importancia botánica y taxonómica, el género ha acaparado gran interés a nivel mundial, debido al valor nutricional que presentan algunas de sus especies, mismas que son fuente importante de alimento como verdura y grano (Wesche-Ebeling et al., 1995). Dentro del género se encuentran plantas silvestres, semidomesticadas y domesticadas productoras de grano, que han contribuido en los procesos de diversificación debido al flujo génico entre ellas (Boege, 2009).

Para la identificación de especies dentro del género Amaranthus se han establecido caracteres reproductivos y vegetativos (Sauer, 1967; Walton, 1968). Entre los rasgos reproductivos de valor taxonómico se encuentran la inflorescencia, flores estaminadas, flores pistiladas e incluso el fruto. En términos generales, la identificación de sus especies se basa en características de las flores pistiladas debido a que presentan la mayoría de los caracteres diagnósticos (Sauer, 1955). Tucker y Sauer (1958) propusieron como caracteres los relacionados con tépalos (tamaño, forma del ápice y dirección), brácteas (tamaño y longitud de la vena media), frutos (base de las ramas del estilo y dirección de los estigmas) e inflorescencias (grosor, hábito, tamaño y densidad en flores). lamonico (2012) señala qué caracteres morfológicos y cualitativos, entre ellos la estructura de la sinflorescencia, la longitud de la sinflorescencia terminal, y la relación entre bráctea-tépalo, permiten reconocer las distintas subunidades reconocibles como taxa. Respecto a la descripción morfológica de estructuras de importancia taxonómica para la identificación de $A$. hybridus L. y $A$. cruentus L. en el sureste de México (desde el estado de Chiapas, Tabasco, la Península de Yucatán), Belice, y Guatemala (zona centro y norte), Solís-Fernández et al. (2020) señalan para $A$. hybridus un largo de bráctea de 2.42-4.75 $\mathrm{mm}$, largo del sépalo externo de 1.65-3.52 mm, y largo del sépalo interno de 1.19-2.58 mm; y en $A$. cruentus brácteas con largo de 1.69-3.39 mm, sépalos externos de 1.89-2.86 $\mathrm{mm}$ de largo, y sépalos internos de 1.16-1.91 mm de largo.

Respecto al origen de especies con valor alimenticio en el continente americano, evidencia antropológica señala que Mesoamérica fue una de las principales regiones de domesticación y comienzo de la agricultura (Kantún-Balam et al., 2013). Una de las primeras civilizaciones que se desarrollaron en esta área fue la Maya, la cual abarcó una gran extensión territorial que comprendió los países de Guatemala, Belice, El Salvador, la porción occidental de Honduras y, en México, los estados de Campeche, Chiapas, Tabasco, Quintana Roo y Yucatán (Ruz-Lhuillier, 1981; Drew, 2002). Esta civilización se caracterizó por el manejo de los recursos naturales, desarrollando diversos sistemas de producción agroforestal, ejemplo de ellos la milpa y el huerto familiar (Méndez-Ramón, 2007). Resultado de lo anterior, se desarrolló una rica y compleja agricultura con varias especies importantes para su alimentación, basada principalmente en maíz (Zea mays L.), frijol (Phaseolus sp.), calabaza (Cucurbita sp.), amaranto (Amaranthus sp.) y otras especies complementarias como cacao (Theobroma cacao L.), vainiIla (Vanilla sp.) y chiles (Capsicum sp.), además de árboles frutales, hierbas y hongos (FAO, 2009).

Con respecto a la domesticación del amaranto, se cuenta con evidencia arqueológica que señala a México como uno de sus centros de origen y domesticación (Sauer, 1950). Por otro lado, Peralta (2009) sugiere que este proceso se pudo llevar a cabo en tres sitios: América del Norte (México), América Central (Guatemala) y América del Sur (Ecuador, Perú y Bolivia). En América tres especies son cultivadas como especie de grano, Amaranthus caudatus L. en los Andes de Perú, $A$. hypochondriacus L. en la región central de México (Peralta, 2009), y A. cruentus en el sur de México y Guatemala (Sauer, 1967), en la región Maya. Incluso en la zona centro de México su grano es usado como 
materia prima para la industria de dulces tradicionales como "la alegría" (Ayala et al., 2014). A pesar de ello, los reportes de la distribución de $A$. cruentus en el sureste de México son escasos, tan solo se ha reportado su presencia en el estado de Chiapas (Espitia-Rangel et. al, 2010b).

Sobre el origen de las especies cultivadas de amaranto, Sauer (1967) propuso dos hipótesis: la primera presenta a Amaranthus hybridus como ancestro común de todas ellas, mientras en la segunda, considera que cada especie procede de una especie silvestre donde $A$. caudatus deriva de $A$. quitensis Kunth, $A$. cruentus de $A$. hybridus y $A$. hypochondriacus de $A$. powellii S. Watson. Estudios a nivel molecular del ADN de $A$. hybridus y especies de amaranto productoras de grano usando marcadores RAPD e isoenzimas (Chan y Sun, 1997), microsatélites (Kietlinski et al., 2014), y SNP o Polimorfismo de Nucleotidos Unicos (Clouse et al., 2016) respaldan las afirmaciones anteriores, al señalar a $A$. hybridus como el ancestro común de las especies de grano de amaranto. Las especies cultivadas empleadas para la producción de grano son fácilmente reconocidas por presentar brácteas cortas y frágiles, inflorescencias de gran tamaño y semillas blancas, resultado de la selección artificial; a diferencia de las silvestres que presentan brácteas espinosas, tallas bajas de plantas e inflorescencias, reducida producción de semillas y semillas oscuras (Sauer, 1967); aunque el color oscuro puede observarse también en organismos semidomesticados o en proceso de domesticación (Stetter et al., 2019).

Sobre la diferenciación entre las especies cultivadas de grano, la morfología de la bractéola es uno de los pocos caracteres que ayudan en su identificación, especialmente entre $A$. caudatus y $A$. cruentus, e incluso entre sus parientes silvestres como A. hybridus, A. quitensis y A. powellii. De esta forma las brácteas en los amarantos de grano son menos espinosas y más cortas y delgadas que las de sus parientes silvestres (Sauer, 1950; Espitia-Rangel et al., 2010c).

Partiendo de la evidencia del origen de A. cruentus, su cercanía con culturas originarias de América, su importancia con estas culturas y las hipótesis que señalan a $A$. hybridus como su ancestro durante el proceso de domesticación y su pariente silvestre más cercano, el presente trabajo tiene como objetivo presentar evidencia sobre la distribución actualizada y la frecuencia de $A$. cruentus y $A$. hybridus, así como evaluar la diversidad morfológica de ambas especies tomando como base caracteres de flores pistiladas y del fruto.

\section{Materiales y Métodos}

\section{Especímenes evaluados y reporte de distribución}

Se utilizaron para el estudio un total de 147 ejemplares procedentes de los herbarios CAS, CICY, ENCB, F, MEXU, MO y NHM (acrónimos con base en Thiers, 2020+ continuamente actualizado), señalados con colectas en Guatemala, Honduras y México, para las especies Amaranthus cruentus y $A$. hybridus. Estos ejemplares fueron reportados como individuos de las especies $A$. cruentus y $A$. hybridus, identificados usando métodos de botánica tradicional, análisis de varianza permutacional multivariada PERMANOVA (por sus siglas en inglés, permutational multivariate variance analysis) y la prueba de porcentaje de similaridad SIMPER (por sus siglas en inglés; similarity percentage) mediante el uso de la paquetería estadística PRIMER v. 7 y PERMANOVA por Solís-Fernández et al. (2020).

Se actualizó la información de distribución y frecuencia de A. cruentus y A. hybridus en Guatemala, Honduras y el sureste de México; el reporte de su distribución extendida se realizó con la información recabada de las etiquetas de los ejemplares de herbario.

\section{Estudio morfológico}

El estudio de diversidad morfológica realizado en $A$. cruentus y $A$. hybridus se basó en la terminología y los 22 caracteres morfológicos (Cuadros 1, 2), reportados por Solís-Fernández et al. (2020), usando para ello imágenes digitales obtenidas a través de un microscopio estereoscopico Nikon (modelo SMZ745T, Tokio, Japón), cámara digital INFINITY1 (modelo 3C, Ontario, Canadá) y el programa INFINITY ANALYZE v. 5.0.3 software (Lumenera Corp., 2010).

\section{Análisis de datos}

La diversidad morfológica se determinó mediante la morfometría de las estructuras florales pistiladas y el fruto, realizando análisis de estadística descriptiva y la determinación del coeficiente de variación. Se aplicó a esta misma información un análisis multivariado de componentes principales (CP). Para obtener la diversidad morfológica, los 
Cuadro 1: Estadística descriptiva entre caracteres morfológicos relacionados a flores pistiladas de Amaranthus cruentus L. procedentes de Guatemala y México y el valor general de ambas procedencias usados comúnmente en la determinación taxonómica de la familia Amaranthaceae. Coeficiente de variación=CV, Largo de la Flor=LF, Largo de la Bráctea=LB, Ancho de la Bráctea=AB, Largo del Sépalo Externo=LSE, Ancho del Sépalo Externo=ASE, Largo del Ápice del Sépalo Externo=LASE, Largo de la Lámina del Sépalo Externo=LLSE, Índice del Sépalo Externo=ISE, Largo del Sépalo Interno=LSI, Ancho del Sépalo Interno=ASI, Largo del Ápice del Sépalo Interno=LASI, Largo de la Lámina del Sépalo Interno=LLSI, Índice del Sépalo Interno=ISI, Largo del Estilo=LE, Largo del Utrículo=LU, Ancho del Utrículo=AU, Relación entre el Largo con respecto a el Ancho del Utrículo=RLAU, Largo de la Semilla=LS, Ancho de la Semilla=AS, Relación entre el Largo de la Bráctea con respecto al largo del Sépalo Externo=RLBSE, Relación entre el Largo del Utrículo con respecto a el largo del Sépalo Externo=RLUSE, Relación entre el Largo de la Bráctea con respecto a el largo del Utrículo=RLBU.

\begin{tabular}{|c|c|c|c|c|c|c|c|c|c|c|c|c|}
\hline \multirow[b]{2}{*}{ Carácter } & \multicolumn{4}{|c|}{ Guatemala } & \multicolumn{4}{|c|}{ México } & \multicolumn{4}{|c|}{ General } \\
\hline & $\begin{array}{l}\text { Media } \\
(\mathrm{mm})\end{array}$ & $\begin{array}{c}\text { Desviación } \\
\text { estándar }\end{array}$ & Varianza & $\begin{array}{l}\text { CV } \\
(\%)\end{array}$ & $\begin{array}{l}\text { Media } \\
(\mathrm{mm})\end{array}$ & $\begin{array}{c}\text { Desviación } \\
\text { estándar }\end{array}$ & Varianza & $\begin{array}{l}\mathrm{CV} \\
(\%)\end{array}$ & $\begin{array}{c}\text { Media } \\
(\mathrm{mm})\end{array}$ & $\begin{array}{c}\text { Desviación } \\
\text { estándar }\end{array}$ & Varianza & $\begin{array}{l}\mathrm{CV} \\
(\%)\end{array}$ \\
\hline LF & 1.97 & 0.46 & 0.21 & 23.30 & 1.49 & 0.35 & 0.12 & 23.33 & 1.90 & 0.47 & 0.22 & 24.52 \\
\hline LB & 2.37 & 0.45 & 0.20 & 19.06 & 2.21 & 0.56 & 0.32 & 25.41 & 2.35 & 0.45 & 0.20 & 19.07 \\
\hline$A B$ & 0.49 & 0.22 & 0.05 & 43.77 & 0.43 & 0.09 & 0.01 & 21.38 & 0.48 & 0.20 & 0.04 & 41.78 \\
\hline LSE & 2.22 & 0.35 & 0.12 & 15.58 & 2.07 & 0.22 & 0.05 & 10.62 & 2.20 & 0.33 & 0.11 & 15.01 \\
\hline ASE & 0.58 & 0.14 & 0.02 & 23.54 & 0.68 & 0.13 & 0.02 & 18.72 & 0.59 & 0.14 & 0.02 & 22.89 \\
\hline LASE & 0.25 & 0.12 & 0.01 & 47.43 & 0.13 & 0.06 & 0.00 & 43.51 & 0.23 & 0.12 & 0.01 & 50.49 \\
\hline LLSE & 1.98 & 0.27 & 0.07 & 13.64 & 1.94 & 0.16 & 0.03 & 8.40 & 1.97 & 0.25 & 0.06 & 12.87 \\
\hline ISE & 4.14 & 1.60 & 2.57 & 38.80 & 3.06 & 0.25 & 0.06 & 8.18 & 3.99 & 1.53 & 2.35 & 38.44 \\
\hline LSI & 1.55 & 0.24 & 0.06 & 15.22 & 1.66 & 0.22 & 0.05 & 13.24 & 1.56 & 0.23 & 0.05 & 14.67 \\
\hline ASI & 0.52 & 0.12 & 0.01 & 22.76 & 0.39 & 0.06 & 0.00 & 14.50 & 0.51 & 0.12 & 0.01 & 23.90 \\
\hline LASI & 0.10 & 0.07 & 0.00 & 68.93 & 0.09 & 0.03 & 0.00 & 31.43 & 0.10 & 0.06 & 0.00 & 64.89 \\
\hline LLSI & 1.45 & 0.27 & 0.07 & 18.59 & 1.57 & 0.19 & 0.04 & 12.20 & 1.46 & 0.26 & 0.07 & 17.60 \\
\hline ISI & 3.12 & 1.01 & 1.02 & 32.30 & 4.33 & 1.19 & 1.42 & 27.49 & 3.28 & 1.07 & 1.15 & 32.71 \\
\hline LE & 0.58 & 0.24 & 0.06 & 40.46 & 0.67 & 0.11 & 0.01 & 15.95 & 0.59 & 0.22 & 0.05 & 37.40 \\
\hline LU & 1.63 & 0.24 & 0.06 & 14.81 & 1.64 & 0.03 & 0.00 & 1.72 & 1.63 & 0.22 & 0.05 & 13.71 \\
\hline$A U$ & 1.35 & 0.25 & 0.06 & 18.65 & 1.24 & 0.27 & 0.07 & 21.67 & 1.33 & 0.25 & 0.06 & 18.47 \\
\hline RLAU & 1.23 & 0.19 & 0.03 & 15.06 & 1.36 & 0.32 & 0.10 & 23.35 & 1.25 & 0.20 & 0.04 & 15.72 \\
\hline LS & 1.17 & 0.17 & 0.03 & 14.53 & 1.16 & 0.10 & 0.01 & 8.53 & 1.17 & 0.16 & 0.03 & 13.66 \\
\hline AS & 1.07 & 0.18 & 0.03 & 16.77 & 1.14 & 0.21 & 0.04 & 18.07 & 1.08 & 0.18 & 0.03 & 16.37 \\
\hline RLBSE & 1.30 & 0.22 & 0.05 & 16.90 & 1.30 & 0.20 & 0.04 & 15.39 & 1.30 & 0.21 & 0.04 & 16.17 \\
\hline RLUSE & 1.07 & 0.20 & 0.04 & 18.59 & 1.21 & 0.01 & 0.00 & 0.65 & 1.09 & 0.19 & 0.04 & 17.49 \\
\hline RLBU & 1.26 & 0.40 & 0.16 & 32.01 & 1.08 & 0.17 & 0.03 & 16.03 & 1.24 & 0.38 & 0.15 & 30.93 \\
\hline
\end{tabular}

caracteres fueron normalizados (matriz de varianza) y se aplicó el coeficiente de distancia euclideana para su representación gráfica. El análisis multivariado se desarrolló con el paquete estadístico PRIMER v. 6 (Clarke y Gorley, 2006).

\section{Resultados}

Distribución y frecuencia de A. cruentus y A. hybridus

El Cuadro 3 presenta la distribución y frecuencia de ejemplares de A. cruentus y A. hybridus por país. Amaranthus cruentus (34 ejemplares en total) se distribuyó en Guatemala (27 ejemplares, 79\%) en siete departamentos, la mayor cantidad en Baja Verapaz y Sacatepéquez, con nueve y ocho ejemplares respectivamente, así como en el sureste de México (siete ejemplares, 21\%), de los cuales tres se ubican en Chiapas, tres en Yucatán y uno en Tabasco.

Por otro lado, Amaranthus hybridus (113 ejemplares en total) se encontró en el sureste de México (55 ejemplares, 49\%); en Guatemala (43 ejemplares, 38\%) donde la mayor cantidad de ejemplares fueron reportados en el departamento de Sacatepéquez (11) y los 32 restantes se distribuyeron en otros 12 departamentos; y Honduras (15 ejemplares, 13\%), principalmente en el departamento de Francisco Morazán, con un total de 12 ejemplares, mientras que los restantes fueron colectados en Comayagua, El Paraíso e Intibucá (Cuadro 3). En México, la mayor cantidad 
Cuadro 2: Estadística descriptiva entre caracteres morfológicos relacionados a flores pistiladas de Amaranthus hybridus L. procedentes de Guatemala y México y el valor general de ambas procedencias usados comúnmente en la determinación taxonómica de la familia Amaranthaceae. Coeficiente de variación=CV, Largo de la Flor=LF, Largo de la Bráctea=LB, Ancho de la Bráctea=AB, Largo del Sépalo Externo=LSE, Ancho del Sépalo Externo=ASE, Largo del Ápice del Sépalo Externo=LASE, Largo de la Lámina del Sépalo Externo=LLSE, Índice del Sépalo Externo=ISE, Largo del Sépalo Interno=LSI, Ancho del Sépalo Interno=ASI, Largo del Ápice del Sépalo Interno=LASI, Largo de la Lámina del Sépalo Interno=LLSI, Índice del Sépalo Interno=ISI, Largo del Estilo=LE, Largo del Utrículo=LU, Ancho del Utrículo=AU, Relación entre el Largo con respecto al Ancho del Utrículo=RLAU, Largo de la Semilla=LS, Ancho de la Semilla=AS, Relación entre el Largo de la Bráctea con respecto al largo del Sépalo Externo=RLBSE, Relación entre el Largo del Utrículo con respecto a el largo del Sépalo Externo=RLUSE, Relación entre el Largo de la Bráctea con respecto a el largo del Utrículo=RLBU.

\begin{tabular}{|c|c|c|c|c|c|c|c|c|c|c|c|c|}
\hline \multirow[b]{2}{*}{ Carácter } & \multicolumn{4}{|c|}{ Guatemala } & \multicolumn{4}{|c|}{ México } & \multicolumn{4}{|c|}{ General } \\
\hline & $\begin{array}{c}\text { Media } \\
(\mathrm{mm})\end{array}$ & $\begin{array}{c}\text { Desviación } \\
\text { estandar }\end{array}$ & Varianza & $\begin{array}{l}\mathrm{CV} \\
(\%)\end{array}$ & $\begin{array}{c}\text { Media } \\
(\mathrm{mm})\end{array}$ & $\begin{array}{c}\text { Desviación } \\
\text { estandar }\end{array}$ & Varianza & $\begin{array}{l}C V \\
(\%)\end{array}$ & $\begin{array}{c}\text { Media } \\
(\mathrm{mm})\end{array}$ & $\begin{array}{c}\text { Desviación } \\
\text { estandar }\end{array}$ & Varianza & $\begin{array}{l}\text { CV } \\
(\%)\end{array}$ \\
\hline LF & 2.28 & 0.48 & 0.23 & 21.27 & 1.94 & 0.25 & 0.06 & 12.62 & 2.23 & 0.47 & 0.22 & 21.11 \\
\hline LB & 3.70 & 0.64 & 0.41 & 17.22 & 3.06 & 0.45 & 0.20 & 14.65 & 3.61 & 0.65 & 0.42 & 17.91 \\
\hline$A B$ & 0.65 & 0.15 & 0.02 & 22.75 & 0.61 & 0.10 & 0.01 & 16.73 & 0.65 & 0.14 & 0.02 & 22.09 \\
\hline LSE & 2.61 & 0.52 & 0.27 & 19.81 & 2.11 & 0.54 & 0.29 & 25.55 & 2.54 & 0.54 & 0.29 & 21.13 \\
\hline ASE & 0.65 & 0.15 & 0.02 & 22.71 & 0.53 & 0.17 & 0.03 & 32.57 & 0.63 & 0.15 & 0.02 & 24.15 \\
\hline LASE & 0.31 & 0.19 & 0.04 & 62.06 & 0.14 & 0.08 & 0.01 & 53.86 & 0.29 & 0.19 & 0.04 & 65.79 \\
\hline LLSE & 2.29 & 0.39 & 0.16 & 17.18 & 1.97 & 0.48 & 0.23 & 24.41 & 2.25 & 0.41 & 0.17 & 18.31 \\
\hline ISE & 4.24 & 1.29 & 1.66 & 30.39 & 4.06 & 0.60 & 0.36 & 14.70 & 4.21 & 1.22 & 1.48 & 28.84 \\
\hline LSI & 1.79 & 0.39 & 0.15 & 21.71 & 1.77 & 0.56 & 0.32 & 31.70 & 1.79 & 0.40 & 0.16 & 22.55 \\
\hline ASI & 0.45 & 0.11 & 0.01 & 24.63 & 0.49 & 0.11 & 0.01 & 22.04 & 0.46 & 0.11 & 0.01 & 24.04 \\
\hline LASI & 0.11 & 0.07 & 0.01 & 63.37 & 0.10 & 0.02 & 0.00 & 21.91 & 0.11 & 0.07 & 0.00 & 60.86 \\
\hline LLSI & 1.67 & 0.35 & 0.12 & 20.92 & 1.68 & 0.55 & 0.30 & 32.90 & 1.68 & 0.37 & 0.14 & 22.09 \\
\hline ISI & 4.20 & 1.52 & 2.31 & 36.25 & 3.58 & 0.48 & 0.23 & 13.50 & 4.12 & 1.44 & 2.07 & 34.98 \\
\hline LE & 0.82 & 0.34 & 0.12 & 41.45 & 0.34 & 0.31 & 0.10 & 92.54 & 0.76 & 0.37 & 0.14 & 48.80 \\
\hline LU & 1.51 & 0.27 & 0.07 & 17.94 & 1.51 & 0.36 & 0.13 & 23.86 & 1.51 & 0.28 & 0.08 & 18.33 \\
\hline$A U$ & 1.25 & 0.17 & 0.03 & 13.66 & 1.32 & 0.17 & 0.03 & 12.78 & 1.26 & 0.17 & 0.03 & 13.45 \\
\hline RLAU & 1.21 & 0.21 & 0.04 & 17.40 & 1.14 & 0.24 & 0.06 & 20.69 & 1.21 & 0.21 & 0.04 & 17.58 \\
\hline LS & 1.13 & 0.15 & 0.02 & 12.97 & 1.12 & 0.18 & 0.03 & 15.89 & 1.13 & 0.15 & 0.02 & 13.08 \\
\hline AS & 1.09 & 0.16 & 0.03 & 14.68 & 1.00 & 0.17 & 0.03 & 16.78 & 1.08 & 0.16 & 0.03 & 14.94 \\
\hline RLBSE & 1.45 & 0.39 & 0.15 & 26.54 & 1.37 & 0.81 & 0.65 & 59.06 & 1.44 & 0.44 & 0.20 & 30.62 \\
\hline RLUSE & 0.83 & 0.17 & 0.03 & 20.05 & 0.95 & 0.11 & 0.01 & 11.51 & 0.84 & 0.16 & 0.03 & 19.36 \\
\hline RLBU & 1.76 & 0.31 & 0.10 & 17.74 & 1.39 & 0.70 & 0.49 & 50.34 & 1.71 & 0.39 & 0.15 & 22.58 \\
\hline
\end{tabular}

de ejemplares corresponde a los estados de Chiapas (28 ejemplares) y Campeche (16), en tanto que los estados de Quintana Roo, Tabasco y Yucatán presentaron los 11 ejemplares restantes.

\section{Diversidad morfológica en Amaranthus cruentus y A. hybridus}

El análisis exploratorio aplicado en estructuras morfológicas de flores pistiladas y frutos en $A$. cruentus reveló que las flores de mayor longitud fueron las provenientes de Gua- temala (LF: $1.97 \mathrm{~mm}$ ). De igual manera, las brácteas fueron más largas y anchas (LB: $2.37 \mathrm{~mm}$ y AB: $0.49 \mathrm{~mm}$ ) (Cuadro 1). En Amaranthus cruentus, el sépalo externo fue de mayor longitud en ejemplares colectados en Guatemala (LSE, LASE, LLSE e ISE), mientras que su ancho (ASE), fue mayor en ejemplares colectados en México (Cuadro 1). El sépalo interno (LSI, LLSI e ISI) fue de mayor tamaño en ejemplares colectados en México (Cuadro 1). El ancho del sépalo interno (ASI) y el largo del ápice del sépalo interno (LASI) fueron de mayor tamaño en ejemplares colectados 
Cuadro 3: Distribución de registros de colecta de ejemplares de Amaranthus cruentus L. y A. hybridus L. en partes de la región del área Maya (suereste de México, Guatemala y Honduras) (acrónimos con base en Thiers, 2020+ continuamente actualizado).

\begin{tabular}{|c|c|c|c|c|}
\hline PÁlS & & Amaranthus cruentus L. & & Amaranthus hybridus L. \\
\hline Depto. / Estado & Cant. & Colector, Núm. de colecta (Herbario) & Cant. & Colector, Núm. de colecta (Herbario) \\
\hline GUATEMALA & 27 & & 43 & \\
\hline Alta Verapaz & 4 & $\begin{array}{l}\text { von Tüerckheim } 8567(\mathrm{~F}) \text {, Standley 90924(F); } \\
\text { Sauer 1265-D (F); Sauer 1265-E (MO) }\end{array}$ & 5 & $\begin{array}{l}\text { Standley 40806, 69273, } 90147 \text { y } 91312 \text { (F); Williams et al. } 25336 \\
\text { (F) }\end{array}$ \\
\hline Baja Verapaz & 9 & $\begin{array}{l}\text { Sánchez-del Pino et al. 520, 521, 523, 524, } \\
526,526-1,526-2,527 \text { y } 530 \text { (CICY) }\end{array}$ & 1 & Sánchez-del Pino et al. 528 (CICY) \\
\hline Chimaltenango & 2 & Saur 1168-K (F); Sauer 1240-B (MO) & 5 & Standley 59043, 59046, 59076, 79743 y 80832 (F) \\
\hline El Progreso & 1 & Juvenal 2304 (F) & 0 & -- \\
\hline Guatemala & 1 & Sauer 1285-D (MO) & 2 & Sánchez-del Pino 518 (CICY); Brenckle 47428 (CAS) \\
\hline Huehuetenango & 0 & -- & 4 & Williams et al. 41273 (F); Standley 82495, 82994 y 82995 (F) \\
\hline Jalapa & 0 & -- & 4 & Standley 76360, 77139 y 77291 (F); Kellerman 7693 (F) \\
\hline Jutiapa & 0 & -- & 2 & Standley 75135 y 75806 (F) \\
\hline El Petén & 0 & -- & 1 & Landell 15787 (MEXU) \\
\hline Quezaltenango & 0 & -- & 4 & Steyermark 34431 (F); Standley 83166, 83500 y 85879 (F) \\
\hline Sacatepéquez & 8 & $\begin{array}{l}\text { Standley 60352, } 63056 \text { y } 64288(\mathrm{~F}) \text {; Sauer } \\
1175-B, 1176-L, 1177-A, 1177-D, 1176 \text { (MO) }\end{array}$ & 11 & $\begin{array}{l}\text { Sánchez-del Pino et al. 517, 517-A y 517-B (CICY); Standley } \\
\text { 63760, 58041, 59853, 59944, 60327, } 63260 \text { y } 64619 \text { (F); Molina } \\
\text { y Molina } 27054 \text { (F) }\end{array}$ \\
\hline Santa Rosa & 0 & -- & 2 & Standley 77864 y 78252 (F) \\
\hline Sololá & 0 & -- & 1 & Williams et al. 25336 (F) \\
\hline Suchitepéquez & 0 & -- & 1 & Standley 88905 (F) \\
\hline Zacapa & 2 & Standley 3955 y 73955 (F) & 0 & -- \\
\hline HONDURAS & 0 & & 15 & \\
\hline Comayagua & 0 & -- & 1 & Standley y J. Chacón 5666 (CAS) \\
\hline El Paraíso & 0 & -- & 1 & Standley 16735 (CAS) \\
\hline $\begin{array}{l}\text { Francisco } \\
\text { Morazán }\end{array}$ & 0 & -- & 12 & $\begin{array}{l}\text { Standley 1660, 5199, } 28485 \text { y } 29505-\text { A (CAS); Standley y Williams } \\
1400 \text { (CAS); Rodríguez } 416 \text { y } 1057 \text { (F); de Belibasis } 53 \text { (MO); } \\
\text { Nichols } 2028 \text { (MO); Rodríguez } 117 \text { y } 159 \text { (MO); Trochez } 217 \\
\text { (MO) }\end{array}$ \\
\hline Intibucá & 0 & -- & 1 & Standley 25600 (CAS) \\
\hline MÉXICO & 7 & & 55 & \\
\hline Campeche & 0 & -- & 16 & $\begin{array}{l}\text { Sánchez-del Pino et al. } 537 \text { (CICY); Álvarez y Sánchez-del Pino } \\
\text { et al. 4067, } 4260 \text { y } 5192 \text { (MEXU); Alvaro } 744 \text { (MEXU); Cabrera } \\
\text { 11703, 14320, } 14411 \text { y } 15977 \text { (MEXU); Martínez 28326, 28690- } \\
\text { A y 29285 (MEXU); Palmillas 999, 999-A y 999-B (MEXU); } \\
\text { Martínez } 2973 \text { (MO) }\end{array}$ \\
\hline Chiapas & 3 & $\begin{array}{l}\text { Sánchez-del Pino et al. } 532 \text { (CICY); Calzada } \\
2869 \text { (ENCB); Breedlove } 14649 \text { (F) }\end{array}$ & 28 & $\begin{array}{l}\text { Sánchez-del Pino et al. } 535 \text { y 535-C (CICY); Breedlove } 6091 \text { (F); } \\
\text { Breedlove } 7423 \text { y } 12785 \text { (MEXU); Calzada } 2768 \text { (MEXU); Durán } \\
546 \text { (MEXU); García } 643 \text { (MEXU); Gómez } 566 \text { (MEXU); Martínez } \\
9078 \text { (MEXU); Martínez y Lambera } 26150 \text { (MEXU); Méndez } \\
4763 \text { y } 6818 \text { (MEXU); Sant 460; Shilom 419, 1570, 1737, 1738, } \\
4497 \text { y } 7468 \text { (MEXU); Sinaca 2258, 2414, 2427, 2414-A y 2414-B } \\
\text { (MEXU); Breedlove } 14732 \text { y } 41524 \text { (MO); Zuil 758 (MO) }\end{array}$ \\
\hline Quintana Roo & 0 & -- & 4 & $\begin{array}{l}\text { Trejo } 118 \text { (CICY); Balam } 451 \text { (CICY); Ucan } 4036 \text { (CICY); Álvarez } \\
10530 \text { (MEXU) }\end{array}$ \\
\hline Tabasco & 1 & Magaña 1468 (ENCB) & 2 & Sol 1125 (MEXU); Magaña 2456 (ENCB) \\
\hline Yucatán & 3 & $\begin{array}{l}\text { Simá y Sánchez-del Pino } 06 \text { (CICY); Simá } 07 \\
\text { (CICY); Espejel } 97 \text { (F) }\end{array}$ & 5 & $\begin{array}{l}\text { Simá y Sánchez-del Pino } 04 \text { (CICY); Darwin y Sundell } 2052 \text { (MO); } \\
\text { Gaumer } 2447 \text { (F); Rivera } 220 \text { (MEXU); Gaumer } 1081 \text { (USCH) }\end{array}$ \\
\hline Total & 34 & & 113 & \\
\hline
\end{tabular}


en Guatemala (Cuadro 1). Los estigmas y utrículos más largos se observaron en ejemplares de México; solamente el ancho del utrículo fue mayor en ejemplares colectados en Guatemala (Cuadro 1). Las semillas colectadas en Guatemala fueron más largas, pero menos anchas que las procedentes de México (Cuadro 1). Tres relaciones fueron consideradas con base en la longitud de bráctea, sépalo externo y utrículo: (1) bráctea:sépalo externo (RLBSE), (2) utrículo:sépalo externo (RLUSE) y (3) bráctea:utrículo (RLBU). Los valores de RLBSE fueron similares en los ejemplares colectados en Guatemala y México, mientras que RLUSE fue mayor en México y RLBU fue mayor en Guatemala (Cuadro 1).

En Amaranthus cruentus el coeficiente de variación (CV) osciló entre $12.87 \%$ (LLSE) y $64.89 \%$ (LASI) (Cuadro 1). En ejemplares de Guatemala la variación del CV más alta se observó en LASI (68.93\%) y la menor en LLSE (13.64\%); para México los valores del CV fluctuaron entre $43.51 \%$ (LASE) y 0.65\% (RLUSE) (Cuadro 1).

En Amaranthus hybridus se observó mayor tamaño en las estructuras de flores pistiladas de ejemplares colectados de Guatemala (Cuadro 2), referente a el largo de la flor (LF: $2.28 \mathrm{~mm}$ ), largo de las brácteas (LB: $3.70 \mathrm{~mm}$ y AB: $0.65 \mathrm{~mm}$ ) y de los sépalos externos (LSE: $2.61 \mathrm{~mm}$, ASE: $0.65 \mathrm{~mm}$, LASE: $0.31 \mathrm{~mm}$, LLSE: $2.29 \mathrm{~mm}$ y ISE: 4.24) (Cuadro 2). Los sépalos internos fueron de menor tamaño en muestras de México (LSI: 1.77 mm y LASI 0.10 mm). En contraste, el LLSI presentó un mayor largo en ejemplares colectados en México (1.68 $\mathrm{mm}$ ); el ISI fue mayor en ejemplares de Guatemala (4.20) (Cuadro 2). El estigma fue más largo en ejemplares de Guatemala $(0.82 \mathrm{~mm})$; el valor del largo del utrículo fue similar en ejemplares colectados en Guatemala y México, mientras que el ancho fue mayor en los colectados en México (1.32 $\mathrm{mm}$ ), la RLAU (1.21) fue mayor en ejemplares colectados en Guatemala (Cuadro 2). Las semillas de Guatemala fueron de mayor tamaño (LS: $1.13 \mathrm{~mm}$ y AS: 1.09), con respecto a las colectadas en México (Cuadro 2). Los caracteres generados en relación con el largo bráctea: sépalo externo (RLBSE: 1.45) y bráctea: utrículo (RLBU: 1.76) fueron mayores en ejemplares procedentes de Guatemala y los ejemplares de México presentaron una mayor relación entre el utrículo: sépalo externo (RLUSE: 0.95) (Cuadro 2).

En Amaranthus hybridus, el coeficiente de variación señaló altas diferencias entre los ejemplares de ambas pro- cedencias. La menor variación fue de $13.08 \%$ para el LS, y la mayor de $65.79 \%$ para LASE. Entre ejemplares de Guatemala el caracter LASI fue el de mayor variación (63.37\%), el menos variante fue el LS (12.97\%) (Cuadro 2). Para México la mayor variación se observó en LE (92.54\%) y la menor en RLUSE (11.51\%) (Cuadro 2).

La variación revelada en $A$. cruentus en los tres primeros componentes principales (CP) fue de $58 \%$. En el CP1 (28\%) fueron asociados a caracteres del sépalo externo (LSE, LASE, ISE y LLSE), sépalo interno (LSI), y la relación del largo de la bráctea:utrículo (RLBU); al CP2 (17\%) se asociaron caracteres relacionados a la semilla (LS y AS), utrículo (AU) y largo del estigma (LE); y al CP3 (13\%) caracteres relacionados al sépalo interno (ISI e ASI) y bráctea (AB) (Cuadro 4).

La variación revelada en $A$. hybridus en los tres primeros CP fue de $58 \%$. El CP1 (33\%) fue asociado a caracteres del sépalo externo (LSE y LLSE), sépalo interno (LSI, LLSI e ISI), semilla (LS) y la relación entre el largo de bráctea:sépalo externo (RLBSE); el CP2 (15\%) se asoció al sépalo externo (ASE e ISE), y sépalo interno (ASI); el CP3 (10\%) al utrículo (AU) y la relación del largo:ancho del utrículo (RLAU) (Cuadro 4).

Tanto en $A$. cruentus como en $A$. hybridus algunas estructuras florales resultaron relevantes, ya que ambas especies compartieron estructuras florales que revelarón alta variación como ASI, AU, ISE, ISI, LLSE, LS, LSE y LSI (Cuadro 4).

El análisis de ordenamiento para los $C P 1$ y 2 , realizados en $A$. cruentus y $A$. hybridus, mostraron un solo grupo en ambas especies sin que la procedencia de colecta fuera un factor de agrupamiento. Entre los ejemplares de $A$. cruentus se separó del grupo uno de los colectados en Baja Verapaz (Fig. 1).

\section{Discusión}

Actualización de la distribución y frecuencia de A. cruentus y A. hybridus en la zona de estudio Basado en los ejemplares de $A$. cruentus procedentes de la República Méxicana y en los resultados de este estudio, se puede afirmar que esta especie posee una mayor distribución con respecto a lo previamente reportado para el sureste del país, donde fue señalada su baja o limitada presencia, ya que básicamente se reportó solo de Chiapas 
Cuadro 4: Detalles del análisis de componentes principales (CP) realizados en Amaranthus cruentus L. y A. hybridus L., señalando para los tres primeros componentes, su variación y los caracteres morfológicos que intervienen en ella.

\begin{tabular}{|c|c|c|c|c|c|c|c|}
\hline \multirow{2}{*}{ Descripción } & \multicolumn{3}{|c|}{ A. cruentus L. } & \multirow{2}{*}{ Descripción } & \multicolumn{3}{|c|}{ A. hybridus L. } \\
\hline & $\mathrm{CP} 1$ & $\mathrm{CP} 2$ & CP3 & & $\mathrm{CP} 1$ & $\mathrm{CP} 2$ & CP3 \\
\hline Valores propios & 6 & 4 & 3 & Valores propios & 7 & 3 & 2 \\
\hline Variación individual (\%) & 28 & 17 & 13 & Variación individual (\%) & 33 & 15 & 10 \\
\hline Variación acumulada (\%) & 28 & 45 & 58 & Variación acumulada (\%) & 33 & 48 & 58 \\
\hline Largo sépalo externo (LSE) & -0.777 & 0.393 & -0.263 & Largo sépalo interno (LSI) & 0.913 & -0.189 & -0.012 \\
\hline Largo ápice sépalo externo (LASE) & -0.770 & 0.330 & -0.109 & Largo sépalo externo (LSE) & 0.883 & 0.204 & 0.153 \\
\hline Índice sépalo externo (ISE) & -0.740 & 0.119 & -0.086 & Largo lámina del sépalo interno (LLSI) & 0.883 & -0.254 & 0.029 \\
\hline Relación largo bráctea utrículo (RLBU) & -0.705 & 0.021 & 0.165 & Largo lámina del sépalo externo (LLSE) & 0.865 & 0.078 & 0.182 \\
\hline Largo sépalo interno (LSI) & -0.683 & 0.254 & 0.242 & $\begin{array}{l}\text { Relación largo bráctea sépalo externo } \\
\text { (RLBSE) }\end{array}$ & -0.687 & 0.098 & -0.439 \\
\hline $\begin{array}{l}\text { Largo lámina del sépalo externo } \\
\text { (LLSE) }\end{array}$ & -0.659 & 0.361 & -0.292 & Índice sépalo interno (ISI) & 0.627 & 0.435 & -0.324 \\
\hline Largo semilla (LS) & 0.340 & 0.845 & 0.221 & Largo semilla (LS) & 0.624 & -0.370 & -0.341 \\
\hline Ancho semilla (AS) & 0.360 & 0.745 & 0.192 & Índice sépalo externo (ISE) & 0.378 & 0.768 & 0.249 \\
\hline Ancho utrículo (AU) & 0.644 & 0.616 & 0.266 & Ancho sépalo externo (ASE) & 0.307 & -0.709 & -0.199 \\
\hline Largo estigma (LE) & 0.010 & 0.527 & -0.088 & Ancho sépalo interno (ASI) & 0.002 & -0.699 & 0.368 \\
\hline Índice sépalo interno (ISI) & -0.185 & -0.318 & 0.786 & Ancho utrículo (AU) & 0.302 & -0.163 & -0.722 \\
\hline Ancho sépalo interno (ASI) & -0.099 & 0.485 & -0.726 & Relación largo ancho del utrículo (RLAU) & 0.143 & -0.297 & 0.498 \\
\hline Ancho de bráctea $(A B)$ & 0.460 & 0.026 & -0.633 & & & & \\
\hline
\end{tabular}

(Espitia-Rangel et al., 2010a; Fig. 2). En este trabajo se evidencia una mayor distribución, se amplia su extensión territorial por el sureste mexicano, específicamente por los estados de Tabasco, Campeche y Yucatán (Fig. 2), pertenecientes a la Provincia Biótica Península de Yucatán (PBPY), misma que abarca la sección sureste de México (Campeche, Quintana Roo, Yucatán y porciones de Chiapas y Tabasco), el norte de Belice y el departamento guatemalteco de El Petén.

No existen registros previos del cultivo comercial de especies de grano de amaranto, partiendo de los reportes del Sistema de Inovación Agroalimentaria y Pesquera (SIAP, 2018) de la Secretaría de Agricultura, Ganadería, DesarroIlo Rural, Pesca y Alimentación (SAGARPA). El estudio etnobotánico realizado por Solís-Fernández (2015) señaló un reducido uso tradicional de $A$. cruentus en la Península de Yucatán, lo que podría ser uno de los factores limitantes de su presencia.

Los ejemplares de herbario incluidos en este estudio son la base para el reporte de la distribución y diversidad morfológica de $A$. cruentus en el área Maya. Estos contie- nen ejemplares silvestres, que históricamente se originaron por procesos de domesticado, y durante la prohibición de su cultivo en la colonia española se volvieron ferales, escaparon de cultivo o han sido conservados por núcleos familiares como herencia cultural. Por lo tanto, los resultados sobre su distribución nos llevan a suponer un posible vínculo derivado de procesos de domesticación, mismo que podría estar asociado a una limitada área de distribución por depender de circunstancias antropogénicas, en comparación con la distribución geográfica más amplia observada en su pariente silvestre, $A$. hybridus. Si bien su distribución es más amplia, al comparar ésta con reportes de A. cruentus en Chiapas, es posible señalar una abundancia mayor de $A$. cruentus en Yucatán y Chiapas, estados que presentan un área de dispersión y abundancia importante para esta especie en el sureste de México.

Espitia-Rangel et al. (2010a, b, c) han señalado la diversidad de $A$. cruentus en la República mexicana. Para América, Adhikary y Pratt (2015) reportaron el análisis de caracteres morfológicos asociados a flores pistiladas y estaminadas mediante técnicas multivariadas usando compo- 


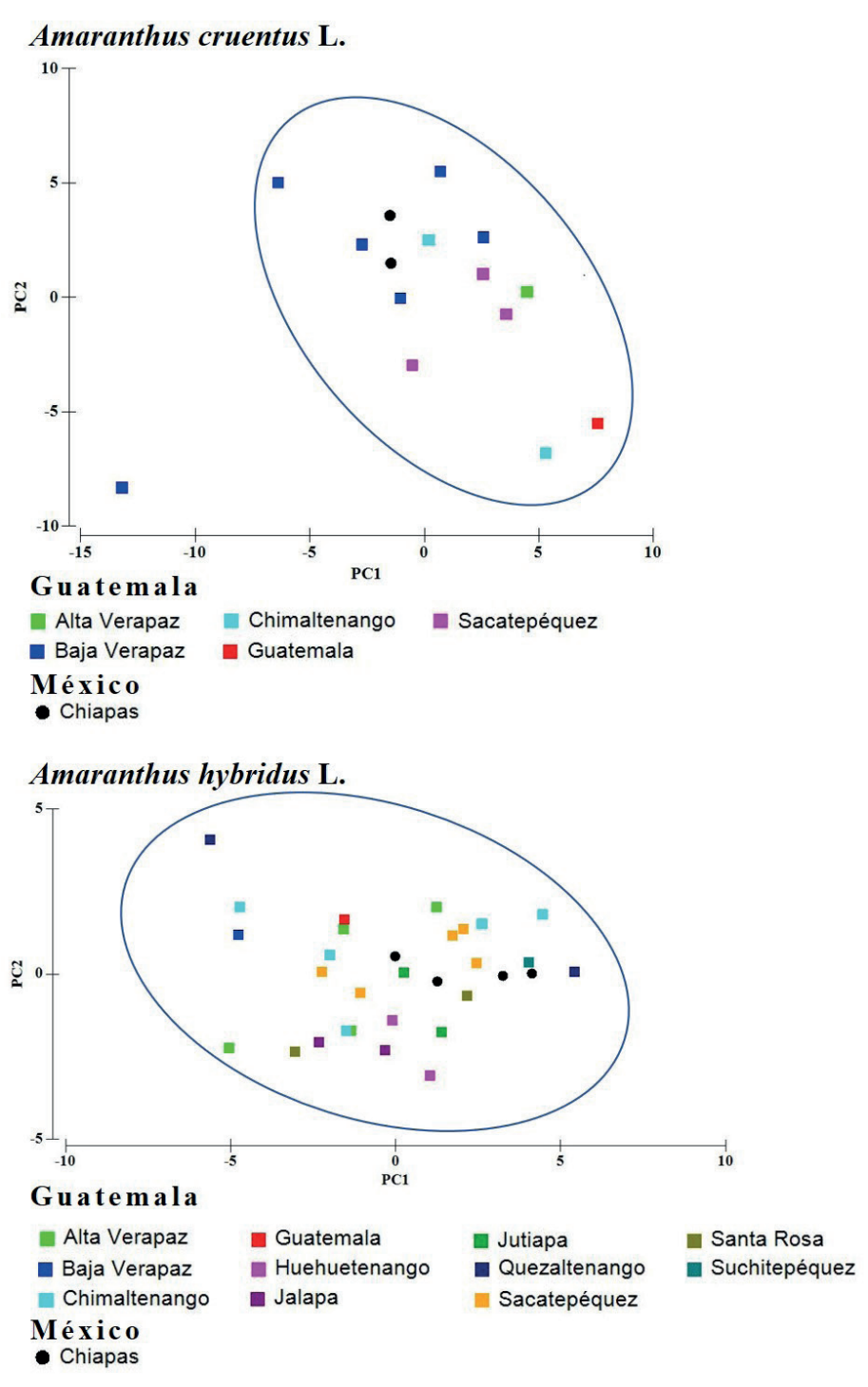

Figura 1: Ordenamiento de Amaranthus cruentus L. y A. hybridus L. con base en el análisis de componentes principales de 22 caracteres morfológicos asociados a las estructuras de flores pistilada con respecto a la procedencia geográfica de colecta de los ejemplares.

nentes principales en especies del complejo Amaranthus hybridus y ejemplares de $A$. cruentus procedentes de México (Sonora y Veracruz), y de A. hybridus de Estados Unidos de América (Indiana y Ohio), México (Puebla y Oaxaca), Guatemala (Huehuetenango y Sacatepéquez), Colombia (Cundinamarca), Perú (Ancash y Apurímac) y Brasil (Goiás). Este último estudio reveló, en $A$. cruentus, que todos los ejemplares se ordenaron en un mismo grupo, observando una variación morfológica continua, una situación similar a lo revelado en el presente trabajo.
Diversidad morfológica entre especies en la zona de estudio

El presente estudio proporciona nueva evidencia respecto a la diversidad en las especies $A$. cruentus y $A$. hybridus, al menos dentro de la región Maya, en la cual podemos observar diferencias morfológicas entre algunos de los ejemplares de $A$. cruentus estudiados, independientemente de su procedencia (Fig. 1).

Las diferencias son significativas en dimensiones del perianto con respecto al fruto y estigma de flores femeninas de $A$. cruentus procedentes de Guatemala con respecto a las presentes en México. El perianto tiene dimensiones mayores en las flores provenientes de Guatemala ( $p$. ej. $\mathrm{LB}=2.37$, $L S E=2.22$, $L L S E=1.98$; Cuadro 1), mientras que las partes reproductivas son mayores en las de México (p. ej. $\mathrm{LE}=0.67$; Cuadro 1). Adicionalmente, este trabajo resalta como uno de los caracteres diagnósticos de relevancia para la identificación de taxa en el género a la relación entre el largo del sépalo externo y el fruto, la cual presenta muy poca variación para los ejemplares de México (p. ej. RLU$S E C V=18.59$, Cuadro 1). Este conjunto de rasgos permite considerar que probablemente las flores de Guatemala han sido menos manejadas en cultivo o domesticación a diferencia de las de México, debido a que los efectos más importantes en los síndromes de domesticación del género es la reducción en la talla de flores y brácteas (Sauer, 1967), así como el incremento en el tamaño del fruto. Además, resulta probable que el tamaño del estigma esté relacionado como una adaptación para la mayor captación de polen (Niklas y Buchmann, 1987; Swanson et al., 2004). Con base en esta posibilidad y sustentando la hipótesis del origen de A. cruentus en Guatemala, el gradiente de distribución observado (Fig. 1) puede ser resultado de la mezcla de formas silvestres, semidomesticadas o ferales que se encuentran en Guatemala, Ilevándose un intercambio genético entre estas formas. Esta suposición se basa en el reporte de Stetter et al. (2019), quienes reportaron, al analizar especies cultivadas de amarantos (A. caudatus, A. cruentus y $A$. hypocondriacus), sus ancestros silvestres ( $A$. hybridus y $A$. quitensis) y organismos semidomesticados, la reducción de talla en caracteres morfológicos asociada al proceso de domesticación. La reducción de talla en este trabajo se observó principalmente en los ejemplares colectados en México, 


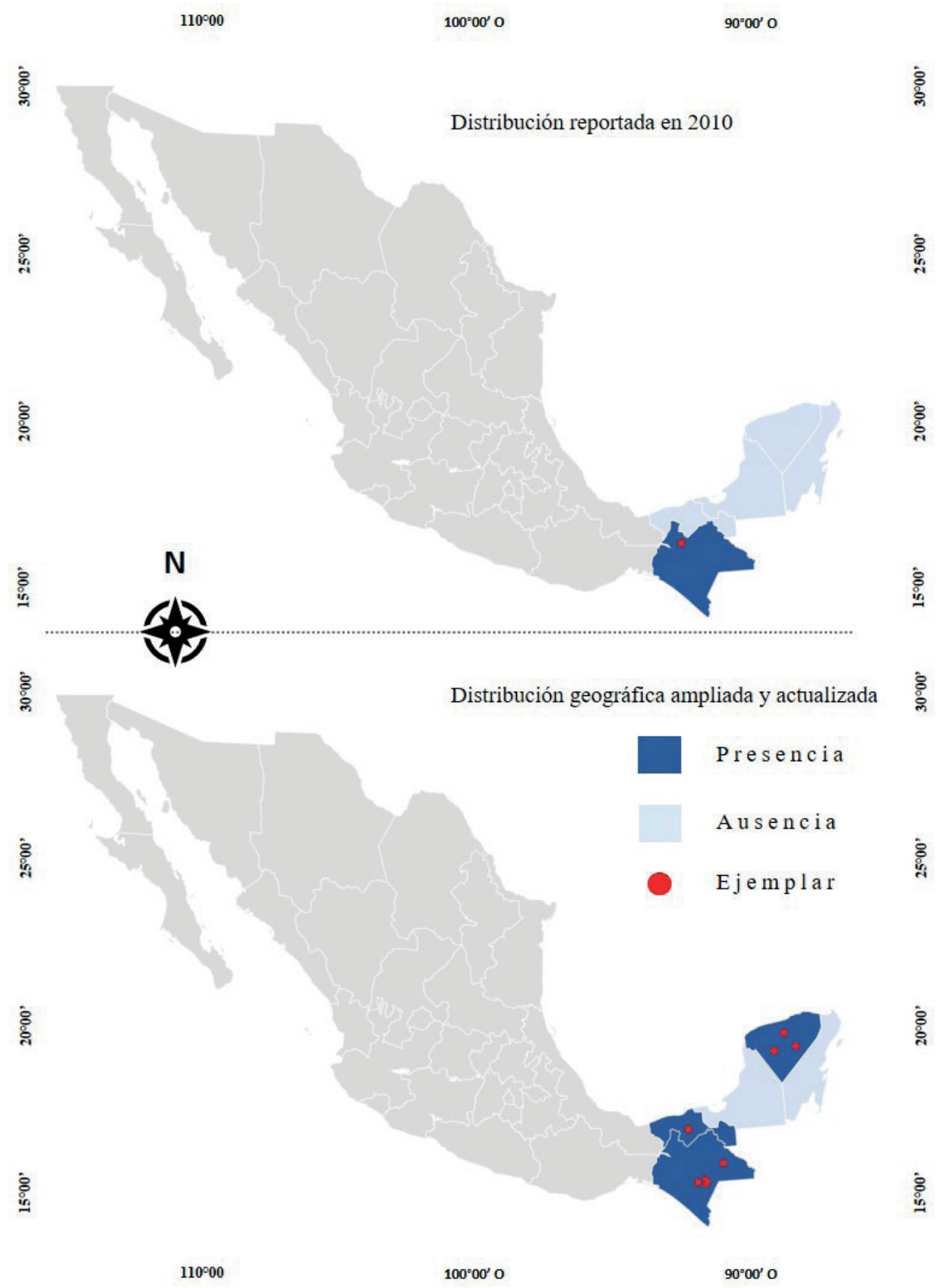

Figura 2: Nuevo reporte sobre la distribución de Amaranthus cruentus L. respecto a su distribución en 2010 para el sureste de México (Campeche, Chiapas, Tabasco, Quintana Roo y Yucatán).

por lo que los procesos de domesticación podrían ser más notorios en está región.

En lo que respecta a $A$. hybridus que es la forma silvestre y maleza, se observaron gradientes de diferenciación morfológica (Cuadro 2) en tamaño de la mayoría de las es- tructuras florales, siendo más evidentes en los especímenes provenientes de Guatemala con respecto a los de México, mientras que el fruto de ambos es similar en tamaño; lo que permite observar un gradiente de diferenciación en la especie (Fig. 1). En este sentido, tan solo se reportan los rangos 
de variación morfológica para la especie contemplando material de la región Maya.

La alta variación morfológica revelada en $A$. cruentus y $A$. hybridus puede ser asociada a una alta diferenciación intraespecífica. Este efecto es reportado en centros de origen y diversificación de las principales especies cultivadas como arroz, maíz, papa y trigo (Boege, 2009). El reporte realizado por Solís-Fernández et al. (2020), sobre la presencia de morfotipos (Amaranthus aff. hybridus-Morphotype 1 y Amaranthus aff. hybridus-Morphotype 2) e híbridos (Amaranthus $\times$ wallichii y Amaranthus hybridus $\times$ Amaranthus cruentus) en la región de colecta, es también una evidencia indirecta de variación morfológica intraespecífica.

Con respecto a la diversidad de $A$. cruentus, Das (2012) reportó un estudio realizado a tres especies de grano del género Amaranthus: dos accesiones de $A$. hypochondriacus, tres de $A$. caudatus y cuatro de $A$. cruentus, así como a especies arvenses del mismo género (A. dubius Mart. ex Thell., $A$. graecizans L., A. hybridus, A. retroflexus L., A. spinosus L. y $A$. viridis $\mathrm{L}$.), analizando la morfología (hojas, flores masculinas y femeninas, semillas y frutos) y bioquímica (compuestos fenólicos y la variación de isoenzimas de la fosfatasa ácida) de las especies incluidas. Como resultado, Das (2012) determinó que el conjunto de caracteres morfológicos y bioquímicos analizados permitieron observar diferencias entre las especies de grano y los arvenses, además de presentar la capacidad de distinguir a $A$. cruentus de las otras dos especies de grano por las características de la inflorescencia (estructura apical, color, grado de ramificación), la semilla (color y ornamentación) y a nivel isoenzimático (perfil). Lo encontrado por Das (2012) se complementa con los resultados obtenidos en el presente estudio sobre la amplia diversidad de $A$. cruentus con respecto a caracteres morfológicos asociados a estructuras florales y esta podría expresarse en características de importancia como la concentración de $\beta$-lainas y proteínas, las cuales son actualmente muy apreciadas en el ramo industrial y de gran valor en la alimentación humana y animal.

Nuestros resultados nos permiten sentar precedentes en la región sureste de México y Guatemala sobre la alta variación morfológica de $A$. cruentus, la cual podría ser una fuente genética de importancia. Espitia-Rangel et al. (2010b) también señalan como importante la región biogeográfica de la Península de Yucatán, aunque discrepamos sobre la observación realizada para esta zona al ser señalada como centro secundario de colecta. Consideramos que se evidencia gran diferenciación morfológica entre ejemplares de esta región, los cuales por distancia geográfica podrían incluir caracteres o alelos diferentes a los encontrados en las áreas con mayor reporte de abundancia como lo ha sido el centro de México, incrementando el acervo genético de $A$. cruentus.

\section{Conclusiones}

Las flores en el género Amaranthus son estructuras básicas para la identificación taxonómica de las especies del grupo. Por lo tanto, la variación morfológica de estas estructuras debe ser baja, para permitir que se conserve la identidad de cada taxon, y en el caso de presentarse gran variación, como hemos observado en este trabajo, suponemos entonces que debe ser un indicador de alta diversidad genética.

Los resultados aquí obtenidos brindan nueva información relacionada a la diversidad genética y distribución geográfica de $A$. cruentus y $A$. hybridus en América, además de presentar evidencia que contribuye a apoyar la hipótesis sobre el centro de domesticación de A. cruentus en Guatemala y su área de diversificación en México. Con base en la caracterización morfológica de flores pistiladas, podemos afirmar alta diversidad genética en $A$. cruentus, incluso mayor a lo observado en $A$. hybridus, situación poco común ya que esta última especie es considerada silvestre. Tomando en cuenta que $A$. cruentus presentó procesos de domesticación relacionados con su importancia para culturas prehispánicas, es posible que aún se presente flujo de genes entre especies, misma que se refleja en la variación morfológica observada. Además, los resultados observados en este trabajo permiten hipotetizar que el germoplasma de $A$. cruentus distribuido en el área Maya podría ser un nuevo acervo genético, al menos diferente al estudiado en la zona centro y pacífico de México, información que contribuye con bases para estudios posteriores de ambas especies para esta región geográfica. Esta hipótesis tambien se sustenta con base en los reportes de distribución y frecuencia presentados en este estudio, que proporcionan evidencia respecto a una distribución extendida de $A$. cruentus dentro de México, misma que se amplía hacia del sureste de México y sobre la cual existen escasos reportes. El reporte de distribución extendida evidencia la 
importancia de contribuir con el incremento del acervo botánico y la conservación de los herbarios como centros de sistematización, representación y conservación de la biodiversidad, al permitir accesar y analizar información histórica y regional. La representatividad de la distribución y frecuencia de una especie con base en registros de herbario está supeditada a la cantidad de ejemplares y la representatividad de su acervo. Los herbarios son depositarios históricos de la biodiversidad de una región o país (Martínez-Castillo y Yánez-Espinosa, 2011), y, por ello, pueden ser tomados como una herramienta básica y fiable en la determinación de distribución y abundancia, tomando mayor valor al estudiar especies representativas del área biogeográfica donde estos se localizan, como es el caso de ambas especies de Amaranthus incluidas en el presente estudio. Partiendo de la idea anterior, este trabajo documenta el acervo botánico de $A$. cruentus en el herbario $\mathrm{CICY}$, aumentando el número de ejemplares a nivel nacional y señalando la presencia de esta especie en la Península de Yucatán, complementando estudios como el realizado por Espitia-Rangel et al. (2010a). En comparación, $A$. hybridus se distribuye prácticamente en todo el territorio nacional, aunque el Eje Volcánico Transversal, el Golfo de México y el Altiplano mexicano son las zonas de mayor distribución.

Con base en lo anterior se sugiere ampliar estudios sobre la diversidad y abundancia de $A$. cruentus en la región sureste de México, extendiendo estos estudios hacia la Península de Yucatán, realizando análisis a nivel molecular, químico, bromatológico y agronómico de $A$. cruentus con la finalidad de revelar su potencial genético, agronómico e industrial.

Se reporta en $A$. hybridus una distribución y frecuencia más amplia que las de $A$. cruentus, quizá debido a que $A$. hybridus es una especie arvense colonizadora, invasiva, tolerante a ambientes adversos y de amplia distribución, asociada a diferentes cultivos a lo largo del mundo (Das, 2016a, b). En contraste, $A$. cruentus es una especie domesticada que, a pesar de haber iniciado este proceso en épocas prehispánicas (Hernández y Herrerías, 1998; Becerra, 2000), su uso como cereal de grano y como verdura fue limitado durante la colonia por razones religiosas de los españoles, al menos en México.

\section{Contribución de autores}

EAC e ISP realizaron la concepción, diseño del estudio, revisión crítica de su contenido intelectual y discusión. RCV contribuyó en la adquisición de datos del material de Guatemala. KSF, AIM e ISP colaboraron en la adquisición de datos y análisis preliminar. EAC e ISP elaboraron el análisis final e interpretación de datos, así como la redacción del documento. EAC, KSF, AIM, RCV e ISP aprobaron el manuscrito final.

\section{Financiamiento}

Este estudio fue apoyado por el Consejo Nacional de Ciencia y Tecnología a través de la convocatoria Ciencia Básica 2014 (243153) y Coordinación de Fundaciones Produce Servicio Nacional de Inspección y Certificación de Semillas (03.04.2013.REC.GEN:AGR.22/C subproject BEI-AMA-13-3) a ISP. KSF recibió una beca del Programa de Iniciación Científica 2013-II y 2014-I del Centro de Investigación Científica de Yucatán. EAC recibió una beca del Consejo Nacional de Ciencia y Tecnología a través de la convocatoria Ciencia Básica 2014 (beca 27715/proyecto 243153).

\section{Agradecimientos}

Los autores agradecemos a los curadores de los herbarios CAS, CEDESU, CICY, F, MEXU, MO, NHS, UCAM y UVAL, al personal del INIFAP y a Hilda Flores Olvera del Instituto de Biología de la Universidad Nacional Autónoma de México (UNAM). Gracias a los dos revisores anónimos por sus valiosos comentarios y contribuciones para el mejoramiento de este manuscrito.

\section{Literatura citada}

Adhikary, D. y D. B. Pratt. 2015. Morphologic and taxonomic analysis of the weedy and cultivated Amaranthus hybridus species complex. Systematic Botany 40(2): 604-610. DOI: https://doi.org/10.1600/036364415X688376

Ayala, A. V., P. Rivas-Valencia, L. Cortes-Espinoza, M. de la O-Olán, D. Escobedo-López y E. Espitia-Rangel. 2014. La rentabilidad del cultivo de amaranto (Amaranthus spp.) en la región centro de México. Ciencia Ergo Sum 21(1): 47-54.

Becerra, R. 2000. El amaranto: Nuevas tecnologías para un antiguo cultivo. Biodiversitas 30(5): 1-6. 
Boege, S. 2009. Centros de origen, pueblos indígenas y diversificación del maíz. Ciencia 60(1): 18-28.

Borsch, T., P. Hernández-Ledesma, W. G. Berendsohn, H. Flores-Olvera, H. Ochoterena, F. O. Zuloaga, S. von Mering y N. Kilian. 2015. An integrative and dynamic approach for monographing species-rich plant groups-Building the global synthesis of the angiosperm order Caryophyllales. Perspectives in Plant Ecology, Evolution and Systematics 17(4): 284-300. DOI: https://doi.org/10.1016/j.ppees.2015.05.003

Carmona, W. y G. Orsini-Velázquez. 2010. Sinopsis del subgénero Amaranthus (Amaranthus, Amaranthaceae) en Venezuela. Acta Botanica Venezuelica 33(2): 329-356.

Chan, K. F. y M. Sun. 1997. Genetic diversity and relationships detected by isozyme and RAPD analysis of crop and wild species of Amaranthus. Theoretical and Applied Genetics 95: 865873. DOI: https://doi.org/10.1007/s001220050637

Clarke, K. R. y R. N. Gorley. 2006. PRIMER v6: User Manual/Tutorial (Plymouth Routines in Multivariate Ecological Research). PRIMER-E. Plymouth, USA. 190 pp.

Clouse, J. W., D. Adhikary, J. T. Page, T. Ramaraj, M. K. Deyholos, J. A. Udall, D. J. Fairbanks, E. N. Jellen, y P. J. Maughan. 2016. The Amaranth Genome: Genome, Transcriptome, and Physical Map Assembly. The Plant Genome 9(3): 1-14. DOI: https:// doi.org/10.3835/plantgenome2015.07.0062er

Costea, M., A. Sanders y G. Waines. 2001. Preliminary results toward a revision of the Amaranthus hybridus species complex (Amaranthaceae). SIDA, Contributions to Botany 19(4): 931974.

Das, S. 2012. Taxonomical observation on the grain amaranths and new varieties of Amaranthus cruentus (Amaranthaceae). Nordic Journal of Botany 30(4): 412-420. DOI: https://doi. org/10.1111/j.1756-1051.2011.01383.x

Das, S. 2016a. Amarants: The crop of great prospect. In: Das, S. (ed.). Amaranthus: A promising crop of future. Spring Science+Business Media. Singapore, Singapore. Pp. 13-48. DOI: https://doi.org/10.1007/978-981-10-1469-7_3

Das, S. 2016b. Weed and herbicide resistance. In: Das, S. (ed.). Amaranthus: A promising crop of future, Spring Science+Business Media. Singapore. Pp. 95-98. DOI: https://doi. org/10.1007/978-981-10-1469-7_3

Drew, D. 2002. Las crónicas perdidas de los reyes Mayas. Colección América Nuestra. Ed. Siglo Veintiuno. México, D.F., México. $500 \mathrm{pp}$.
Espitia-Rangel, E., D. Escobedo-López, E. C. Mapes-Sánchez y C. A. Núñez-Colín. 2010a. Priority areas to collect Amaranthus germplasm in Mexico based on diversity and species richness. Revista Mexicana de Ciencias Agrícolas 1(4): 609-617.

Espitia-Rangel, E., E. C. Mapes-Sánchez, C. A. Nuñez-Colín y D. Escobedo-López. 2010b. Distribución geográfica de las especies cultivadas de Amaranthus y sus parientes silvestres en México. Revista Mexicana de Ciencias Agrícolas 1(3): 609-617.

Espitia-Rangel, E., E. C. Mapes-Sánchez, D. Escobedo-López, M. de la O.-Olán, P. Rivas-Valencia, G. Martínez-Trejo, L. CórtesEspinosa y J. M. Hernández-Casillas. 2010c. Conservación y uso de los recursos genéticos de Amaranto en México. Instituto Nacional de Investigación Forestales, Agrícolas y Pecuarias, Centro de Investigación Regional Centro. Celaya, México. 200 pp.

FAO. 2009. La FAO en México más de 60 años de cooperación 1945-2009. Organización de las Naciones Unidas para la Agricultura y la Alimentación. México, D.F., México. 340 pp.

Hernández, G. y D. G. Herrerías. 1998. Amaranto: historia y promesa. Ed. Patrimonio histórico de Tehuacán, A.C. México, D.F., México. 529 pp.

lamonico, D. 2012. Amaranthus powellii subsp. cacciatoi comb. et stat. nov. (Amaranthaceae). Nordic Journal of Botany 30(1): 12-16. DOI: https://doi.org/10.1111/j.17561051.2011.01080.x

Kantún-Balam, J., J. S. Flores, J. Tun-Garrido, J. Navarro-Alberto, L. Arias-Reyes y J. Martíne-Castillo. 2013. Diversidad y origen geográfico del recurso vegetal en los huertos familiares de Quintana Roo, México. Polibotánica 36: 163-196.

Kietlinski, K. D., F. Jimenez, E. N. Jellen, P. J. Maughan, S. M. Smith y D. B. Pratt. 2014. Relationships between the Weedy Amaranthus hybridus (Amaranthaceae) and the Grain Amaranths. Crop Science 54(1): 220-228. DOI: https://doi. org/10.2135/cropsci2013.03.0173

Lumenera Corp. 2010. Infinity Analyse software version 5.0.3. Ottawa, Canada.

Martínez-Castillo, M. del R. y L. Yáñez-Espinosa. 2011. Herbarios: una mirada crítica. Ciencia y Desarrollo 237(252): 64-69.

Mazón, N., E. Peralta, M. Rivera, C. Subía y C. Tapia. 2003. Catálogo del banco de germoplasma de amaranto (Amaranthus spp.) del INIAP-Ecuador. Programa Nacional de Leguminosas y Granos Andinos, Departamento Nacional de Recursos 
Fitogenéticos y Biotecnología. Estación experimental Santa Catalina. Instituto Nacional de Investigaciones Aagropecuarias. Quito, Ecuador. 98 pp.

Méndez-Rámon, M. 2007. El huerto familiar del Sureste de México. El Colegio de la Frontera Sur. Villahermosa, Tabasco, México. Pp. 19-25.

Mosyakin, S. L. y K. R. Robertson. 1996. New infrageneric taxa and combinations in Amaranthus (Amaranthaceae). Annales Botanici Fennici 33(4): 275-281.

Niklas, K. J. y S. L. Buchmann. 1987. The aerodynamics of pollen capture in two sympatric Ephedra species. Evolution 41(1): 104-123. DOI: https://doi.org/10.1111/j.1558-5646.1987. tb05774.x

Peralta, E. 2009. Amaranto y ataco: Preguntas y respuestas. Instituto Nacional de Investigacón Agropecuarias, Estación Experimental Santa Catalina, Programa Nacional de Leguminosas y Granos Andinos. Quito, Ecuador. Pp. 1-7.

Ruz-Lhuillier, A. 1981. El pueblo Maya. Ed. Salvat Editores. México, D.F., México. 32 pp.

Sauer, J. D. 1950. The grain amaranths: A survey of their history and classification. Annals of the Missouri Botanical Garden 37(4): 561-632. DOI: https://doi.org/10.2307/276425

Sauer, J. D. 1955. Revision of the dioecious amaranths. Madroño 13: 5-46.

Sauer, J. D. 1967. The grain amaranths and their relatives: A revised taxonomic and geographic survey. Annals of the Missouri Botanical Garden. 54(2): 103-137. DOI: https://doi. $\operatorname{org} / 10.2307 / 2394998$

SIAP. 2018. Servicio de Información Agroalimentaria y Pesquera, Anuario Estadístico de la Producción Agrícola. https://nube. siap.gob.mx/cierreagricola/ (consultado mayo de 2020).

Solís-Fernández, K. Z. 2015. Valoración morfológica, usos y distribución del Amaranto (Amaranthus cruentus L. y Amaranthus hybridus L.) en algunas zonas de la región Maya. Te- sis de licenciatura. Instituto Técnológico de Conkal. Conkal, Yucatán, México. 121 pp.

Solís-Fernández, K. Z., E. A. Aguilera-Cauich, R. Cifuentes-Velasquez, H. Flores-Olvera, R. H. Andueza-Noh e I. Sánchez-del Pino. 2020. High morphological diversity of Amaranthus cruentus (Amaranthaceae) and its putative wild ancestor revealed by pistillate floral characterization in the proposed domestication center of origin. Phytotaxa 439(3): 217-230. DOI: https://doi.org/10.11646/phytotaxa.439.3.4

Stetter, G., M. Vidal-Villarejo y K. J. Schmid. 2019. Parallel seed color adaptation during multiple domestication attempts of an ancient new world grain. Molecular Biology and Evolution 37(5): 1407-1419. DOI: https://doi.org/10.1093/mol$\mathrm{bev} / \mathrm{msz304}$

Swanson, R., A. F. Edlund y D. Preuss. 2004. Species specificity in pollen-pistil interactions. Annual Review of Genetics 38: 793-818. DOI: https://doi.org/10.1146/annurev.genet.38.072902.092356

Thiers, B. 2020+. Index herbariorum: A global directory of public herbaria and associated staff. New York Botanical Gardens Virtual Herbarium. http://sweetgum.nybg.org/science/ih/ (consultado abril de 2020).

Tucker, J. M. y J. D. Sauer. 1958. Aberrant Amaranthus population of Sacramento-San Joaquin delta, California. Madroño 14: 252-261.

Walton, P. D. 1968. The use of Amaranthus caudatus in simulating the breeding behavior of commercial Gossypium species. Journal of Heredity 59(1): 17-18. DOI: https://doi. org/10.1093/oxfordjournals.jhered.a107630

Wesche-Ebeling, P., M. Ratikanta, G. García-Díaz, D. I. González y F. Sosa-Alvarado. 1995. Contributions to the Botany and Nutritional Value of Some Wild Amaranthus Species (Amaranthaceae) of Nuevo Leon, Mexico. Economic Botany 49(4): 423-430. DOI: https://doi.org/10.1007/BF02863094 